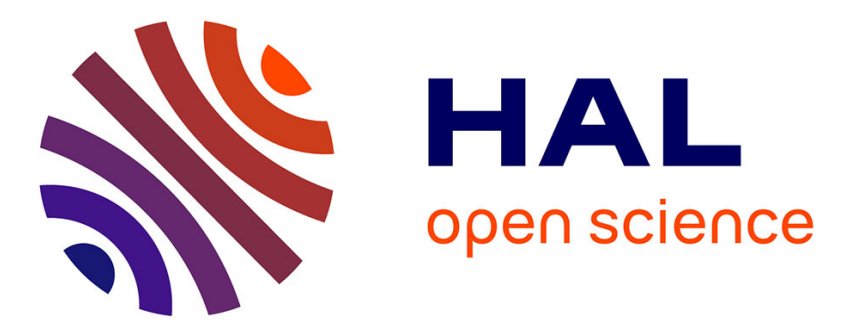

\title{
Laser-induced field-free alignment of OCS molecule
}

\author{
V. Loriot, P. Tzallas, E. P. Benis, E. Hertz, B. Lavorel, D. Charalambidis, O. \\ Faucher
}

\section{To cite this version:}

V. Loriot, P. Tzallas, E. P. Benis, E. Hertz, B. Lavorel, et al.. Laser-induced field-free alignment of OCS molecule. Journal of Physics B: Atomic, Molecular and Optical Physics, 2007, 40, pp.2503. hal-00397122

\section{HAL Id: hal-00397122 \\ https://hal.science/hal-00397122}

Submitted on 19 Jun 2009

HAL is a multi-disciplinary open access archive for the deposit and dissemination of scientific research documents, whether they are published or not. The documents may come from teaching and research institutions in France or abroad, or from public or private research centers.
L'archive ouverte pluridisciplinaire HAL, est destinée au dépôt et à la diffusion de documents scientifiques de niveau recherche, publiés ou non, émanant des établissements d'enseignement et de recherche français ou étrangers, des laboratoires publics ou privés. 


\title{
Laser-induced field-free alignment of the OCS molecule
}

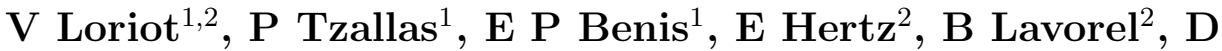 \\ Charalambidis $^{1,3}$ and O Faucher ${ }^{1,2}$ \\ ${ }^{1}$ Foundation for Research and Technology Hellas, Institute of Electronic Structure \\ and Laser, P.O. Box 1527, GR-71110 Heraklion (Crete), Greece \\ ${ }^{2}$ Institut Carnot de Bourgogne, UMR 5209 CNRS-Université de Bourgogne, BP \\ 47870, FR-21078 Dijon Cedex, France \\ ${ }^{3}$ Department of Physics, University of Crete, P.O. Box 2208, GR-71003 Heraklion \\ (Crete), Greece
}

\begin{abstract}
We investigate the dynamical alignment of jet-cooled OCS molecules induced by a short laser pulse. The alignment is measured through the orientational contribution of the optical Kerr effect using a second weak laser pulse as a probe. Maximum alignment is observed at conditions close to saturation of ionization. The results are analysed with a quantum mechanical model solving for the rotational dynamics.
\end{abstract}


Molecular field-free alignment has received enhanced attention (for a review see reference [1]) after its first observation in $\mathrm{I}_{2}$ [2]. In principle, any molecule of anisotropic polarizability kicked by an intense and sufficiently short laser pulse can be aligned in field-free conditions, i.e. after extinction of the pulse. Even molecules that are highly symmetrical in their ground vibronic state (like for instance $\mathrm{CH}_{4}$ ) would be aligned, providing that they are excited in a state of non-spherical symmetry. However, most of the studies have been performed through the non-resonant interaction of the laser field with the ground vibronic state of the molecule [3]. When one uses laser pulses of duration $\tau_{L}$ much longer than the rotational period defined by $T_{r}=1 /(2 B c)$, with $B$ one of the rotational constants and $c$ the speed of light, the alignment is adiabatic and occurs therefore only during the interaction [4]. On the other hand, a short pulse $\tau_{L} \ll T_{r}$ produces post-pulse alignment transients of duration much shorter than the rotational period. For linear molecules, the transients are spaced in time by a fraction of the rotational period. If one considers the rigid rotor approximation, the effect lasts as long as the coherence of the medium is maintained. The main advantage of the short pulse is that applications based on aligned molecules can be conducted in field-free conditions.

Most of the studies devoted to laser-induced alignment of small molecules have been focused on non-polar molecules [1]. In fact, the permanent dipole plays a negligible role when the alignment occurs through the non-resonant excitation of the polarizability. However, alignment of polar molecules is an interesting issue due to its close relation to orientation. Laser-induced orientation has been achieved by combining an electrostatic and a pulsed laser field in the adiabatic regime $[5,6]$. So far, observation of orientation in the short pulse regime [7] remains out of reach. Orientation presents important prospects with respect to control of steric effects in physical chemistry but also to nonlinear optics. In the latter, processes for instance prohibited in centrosymmetric media could be allowed in the gas phase, like the generation of even order harmonics.

The scope of this work is to present a detailed study of field-free alignment of a polar molecule using carbonyl sulfide as a target molecule. The OCS molecule is aligned nonresonantly by a short pump pulse of duration $\sim 70$ fs and intensity $10-20 \mathrm{TW} / \mathrm{cm}^{2}$. The detection is based on the optical Kerr effect. The alignment is probed by using a second pulse that interacts with the molecules after they have been exposed to the pump pulse. The anisotropic angular distribution of the molecular axis results in the depolarization of the probe that can be observed in time by changing the delay between the two pulses. Because the second pulse is weak, we can safely assume that the alignment is not affected by the probe and therefore that the signal reflects only the alignment produced by the first pulse.

The pump and probe pulses are delivered by a Ti:Sapphire laser chirped pulse amplified system operating at $10 \mathrm{~Hz}$ with a central wavelength of $800 \mathrm{~nm}$, delivering pulses with energy up to $150 \mathrm{~mJ}$ and duration down to $50 \mathrm{fs}$. Although we use a vacuum setup designed for the generation of high order harmonics through large interaction volume [8], the scheme of the experiment is similar to the one presented in reference [9]. 
A delay line is used in order to adjust the temporal delay between the pump and probe pulse. Both pulses are linearly polarized at $45^{\circ}$ to each other. For improved alignment efficiency, the molecules are cooled in a jet expansion of pure OCS operating at a backing pressure of 2-4 bars. Both beams are focused with a lens of $3 \mathrm{~m}$ focal length and cross each other at a small angle $\left(\sim 1^{\circ}\right)$ into the jet expansion, close to the nozzle where the number density is large. The jet axis is intersected by the two laser beams at $90^{\circ}$. At the exit of the interaction chamber, the pump is blocked by a beam stop and the depolarization of the probe is detected through a polarizer set at $90^{\circ}$ with respect to its initial polarization direction. The depolarized probe field is then collected with a photomultiplier, sampled by a boxcar integrator, and sent to a computer that is also used to control the delay line.

In the limit of small birefringence, the intensity signal $\mathcal{S}$ measured at the pumpprobe delay $\tau$ is approximated by

$$
\mathcal{S}(\tau) \propto \int_{-\infty}^{+\infty} d t I_{p r}(t-\tau)\left(\Delta n_{O C S}(t)+\mathcal{P}\right)^{2}
$$

where $I_{p r}(t)$ is intensity envelope on the probe pulse, $\Delta n_{O C S}(t)=n_{\|}(t)-n_{\perp}(t)$ is the difference of refractive indices of OCS between the parallel (I) and any perpendicular $(\perp)$ direction of the pump polarization axis, and $\mathcal{P}$ is a parameter that applies when heterodyned detection occurs $[10,11]$. For a linear molecule, it has been shown [12] that

$$
\Delta n_{O C S}(t)=\frac{3 N \Delta \alpha}{4 n \epsilon_{0}}\left(\left\langle\cos ^{2} \theta\right\rangle_{T_{0}}(t)-\frac{1}{3}\right),
$$

with $n$ the average value of the refractive index at the probe frequency, $N$ the number density, $\epsilon_{0}$ the dielectric constant of vacuum, and $\Delta \alpha=4.04 \AA^{3}$ the anisotropy of polarizability for OCS [13]. Higher-order polarizabilities have been neglected. In this expression, $\left\langle\cos ^{2} \theta\right\rangle_{T_{0}}$ defines the quantum and thermally averaged value that characterizes the extent of alignment with $T_{0}$ the initial temperature of the gas sample and $\theta$ the angle between the molecular axis and the polarization direction of the pump electric field. For a randomly oriented ensemble of molecules, we have $\left\langle\cos ^{2} \theta\right\rangle=\frac{1}{3}$ and thus $\Delta n_{O C S}(t)=0$.

Figure 1 (lower panel) displays the signal $\mathcal{S}(\tau)$ measured in pure OCS. The experiment is performed with a pump intensity $I \simeq 20 \mathrm{TW} / \mathrm{cm}^{2}$. Only the signal recorded in the vicinity of the alignment transients is shown. The observation is characterized by transients of field-free alignment corresponding to revivals of the rotational wave packet initially excited by the pump. The revivals are spaced by 41.1 ps that corresponds to $T_{r} / 2$, assuming $B=0.20 \mathrm{~cm}^{-1}$ [14]. Revivals observed at quarter period in molecules with inversion symmetry, where the population is affected by nuclear spin statistics, are not observed in polar molecules $[15,16] .\left\langle\cos ^{2} \theta\right\rangle_{T_{0}}$ is shown in the upper panel of figure 1. It has been obtained by solving the time dependent Schrödinger equation for a thermal ensemble of molecules with the model described in reference [9]. In the simulation, the rotational temperature was adjusted to $T_{0}=100 \mathrm{~K}$ as to provide the best agreement with the observation. This value value is consistent with the experimental conditions assuming an isentropic expansion for the molecular 


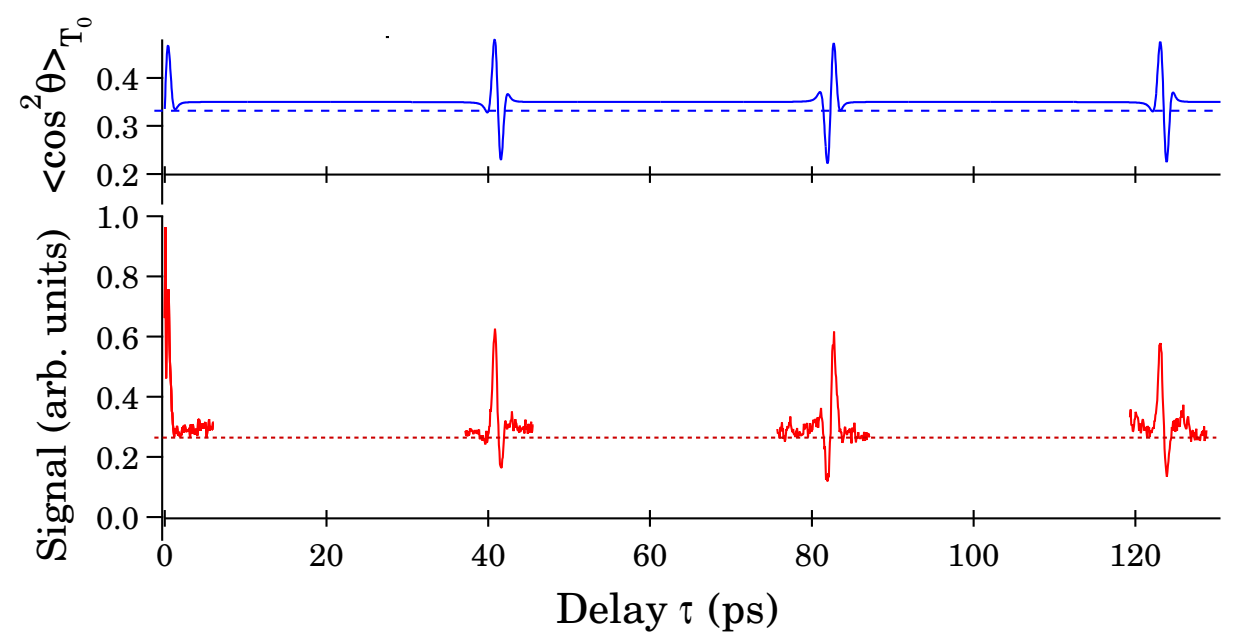

Figure 1. (Color online) Lower panel: heterodyned signal versus pump-probe delay recorded in a supersonic jet of OCS. Upper panel: corresponding value of $\left\langle\cos ^{2} \theta\right\rangle_{T_{0}}$ from numerical simulation (see text).

jet [17]. The dashed line corresponds to $\left\langle\cos ^{2} \theta\right\rangle=1 / 3$. As it has been reported in several studies $[18,12,19]$, the rotational dynamics of a linear molecule leads to a regular alternation of the molecular axis between alignment $\left(\left\langle\cos ^{2} \theta\right\rangle>1 / 3\right)$ and planar delocalization $\left(\left\langle\cos ^{2} \theta\right\rangle<1 / 3\right)$. Between the revivals, the angular distribution remains slightly elongated along the field direction leading to a small permanent alignment with $\left\langle\cos ^{2} \theta\right\rangle \sim 0.35$. The doted line shown in the lower panel indicates the constant signal $\mathcal{S}(\tau) \propto \int_{-\infty}^{+\infty} d t \mathcal{P}^{2} I_{p r}(t-\tau)$ (see equation 1 ) associated with the heterodyne component produced by the probe alone. It originates from uncompensated birefringence from the optical elements, mainly occurring in the windows of the vacuum chamber due to pressure difference. In the present experiment, we can see that the heterodyne component favors the signal on the alignment peaks compared to delocalisation, meaning that the parameter $\mathcal{P}$ introduced in equation 1 is positive.

A thorough analysis of the transient allows for a quantification of the alignment. The structural shape of the revival is governed by the pulse parameters, the temperature, and the probe DC signal when heterodyned detection is used. Figure 2 displays the first revival $\left(T_{r} / 2\right)$ observed at same temperature as in Figure 1, but different pulse intensities $I$ and with a reduced heterodyne component. $I$ is the average intensity deduced from the experimental parameters of the pump beam. The expectation value $\left\langle\cos ^{2} \theta\right\rangle_{T_{0}}$ (upper panel) has been obtained by fitting equation (1) to the experimental data using two free parameters, a scaling factor and the temperature $T_{0}$. The parameter $\mathcal{P}$ was deduced from the DC level after deconvolution by the probe intensity envelope and set as a fixed parameter. The modification of the signal with respect to alignment occurs though the asymmetry of the transients. With homodyne detection, the degree of alignment can be deduced from the asymmetry, the last being mainly due to the intensity-dependent permanent alignment [12]. In the present experiment, where heterodyned detection is 

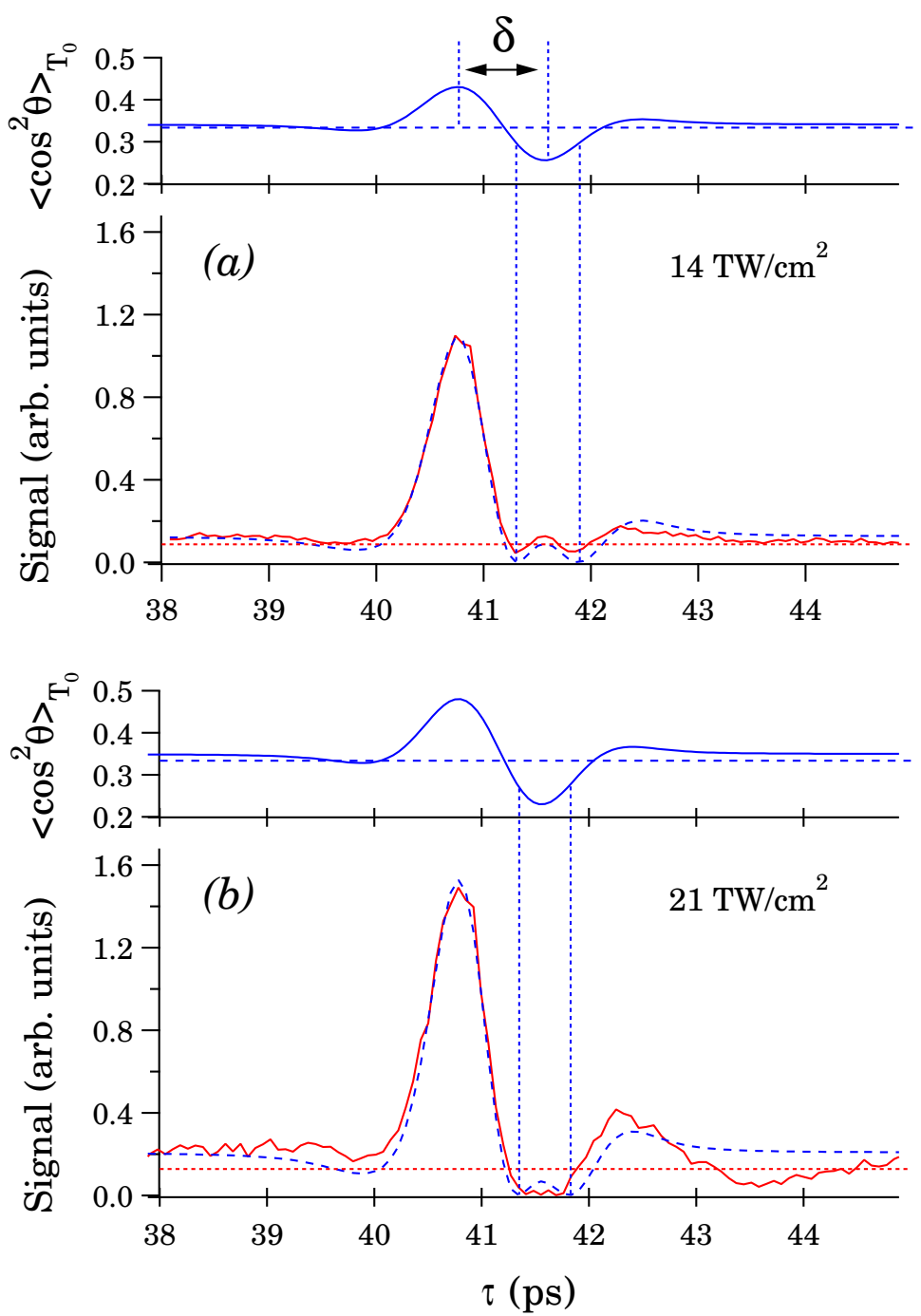

Figure 2. (Color online) Lower panels : Numerical simulations (dashed curves) and heterodyned signals (full curves) recorded for different intensities $I$ of the pump pulse: (a) $14 \mathrm{TW} / \mathrm{cm}^{2}$, (b) $21 \mathrm{TW} / \mathrm{cm}^{2}$. Upper panels : corresponding value of $\left\langle\cos ^{2} \theta\right\rangle_{T_{0}}$ from numerical simulation.

employed, both permanent alignment and heterodyne component happen to contribute with comparable weight to the signal. These contributions can be readily understood from figure 2. The permanent alignment corresponds to the elevation of $\left\langle\cos ^{2} \theta\right\rangle_{T_{0}}$ with respect to the value $1 / 3$. The heterodyne component can be evaluated in terms of $\left\langle\cos ^{2} \theta\right\rangle_{T_{0}}$ just by looking at the delays where the simulated signal drops to zero. For these delays (see the vertical doted lines), the heterodyne component compensates the OCS contribution, i.e. $\Delta n_{O C S} \simeq-\mathcal{P}$, with $\mathcal{P}>0$ in this case. This results in the two minima observed from both sides of the delocalisation peak at $41.6 \mathrm{ps}$.

The signal modification when the pump laser intensity $I$ is increased from $14 \mathrm{TW} / \mathrm{cm}^{2}$ (a) to $21 \mathrm{TW} / \mathrm{cm}^{2}$ (b) is due to the modification of both global alignment (permanent and dynamical) and probe DC signal. The last one occurs because the 
intensity ratio between the pump and probe pulse was kept constant during the experiment. The $50 \%$ increase in the DC level (doted lines) between (a) and (b) reflects therefore directly the change of the probe intensity $I_{p r}$ (see equation 1 ). The alignment peak raising at $\tau=42.4 \mathrm{ps}$ is due to the increasing permanent alignment. Regarding the signal ratio between the two intensities, we should point out the following. For similar experimental conditions, we have shown that $\left\langle\cos ^{2} \theta\right\rangle_{T_{0}}-1 / 3$ varies almost linearly with the pump intensity [20]. More precisely, $\left\langle\cos ^{2} \theta\right\rangle_{T_{0}}-1 / 3$ develops slightly faster (slower) than the intensity on a transient of alignment (delocalisation), because of the permanent alignment. We should therefore expect, in a first approximation (i.e. neglecting heterodyning and permanent alignment), that changing the intensity from 14 to $21 \mathrm{TW} / \mathrm{cm}^{2}$ would result in an increase of the maximum signal by about $(21 / 14)^{3} \simeq 3.3$. Obviously such enhancement is not observed in figure 2. Experiments conducted at larger intensities have revealed no further improvement of the alignment, indicative of saturation through ionization by the pump pulse [9]. The high order harmonics spectra that we have observed in OCS corroborates an upper limit of $30 \mathrm{TW} / \mathrm{cm}^{2}$ for the saturation intensity of ionization (see appendix). Owing to these effects, the maximum alignment achieved in this work was limited, according to the simulation, to $\left\langle\cos ^{2} \theta\right\rangle_{T_{0}} \simeq 0.48$.

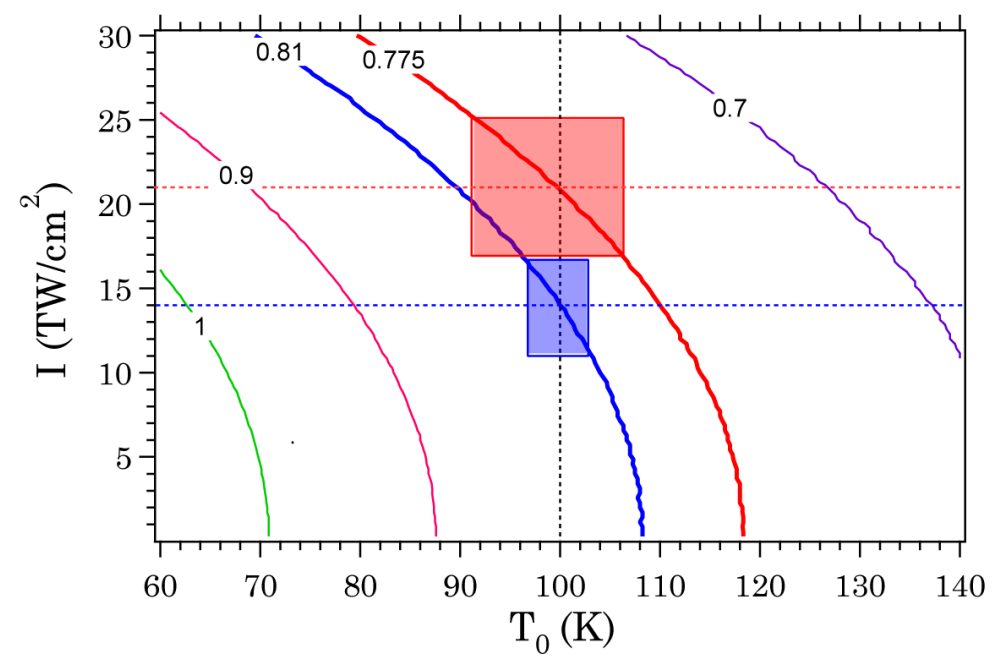

Figure 3. (Color online) Contour plot of the intra-revival temporal time interval $\delta$ (in ps) between the alignment and delocalisation peak calculated for different temperatures $T_{0}$ and intensities $I$. The depicted rectangles represent the uncertainty range of the correlated parameters (see text).

Another interesting issue of this work is the influence of the gas temperature on the recorded signal. The adjusted temperature in figures 1 and 2 is $T_{0}=100 \mathrm{~K}$. As it is known, the degree of alignment improves with lower rotational temperature [21, 22]. For intermediate temperatures, investigated here, a small variation of temperature $( \pm 30 \mathrm{~K})$ mainly affects the width of the transients and the peak ratio between delocalisation and alignment. An estimation of the initial rotational temperature before excitation can 
be inferred from the time interval $\delta$ [depicted in figure $2(\mathrm{a})$ ] between the peaks of alignment and delocalisation. This quantity mirrors the rotational period corresponding to the barycentre of the rotational state distribution that comprises the wave packet after laser excitation [23]. The larger is $\delta$, the lower is the energy of the excited states. However, because the final population does not only depend on the laser intensity $I$ but also on the initial rotational distribution, the width of the revival and therefore $\delta$ is as well affected by the initial temperature $T_{0}$. The correlation between intensity and temperature is illustrated by the simulation of figure 3 , where $\delta$ is depicted versus $T_{0}$ and $I$ for the revival of figure 2. The contour $\delta=0.81$ and 0.775 ps corresponds to the parameters of figure 2 (a) and (b), respectively. One can see for instance that a variation of $\pm 20 \%$ on the intensity of (a) and (b) translates to an uncertainty on the temperature of $\pm 3 \mathrm{~K}$ and $\pm 7 \mathrm{~K}$ (see the depicted rectangles), respectively. These values are about the standard deviations given by the corresponding fits. Considering an experimental intensity known with an uncertainty of $\pm 20 \%$, one can reasonably assume that the temperature was $100 \pm 10 \mathrm{~K}$.

In conclusion, we have shown that the OCS molecule can be aligned in a nonadiabatic regime by a short laser pulse. A comparison between the experimental data and calculations based on a rotational dynamics quantum mechanical model allowed for estimations of the degree of alignment. The maximum alignment was observed to be limited by saturation of ionization. In order to increase the alignment beyond the limit imposed by ionization, the laser pulse should be optimized. One possibility would be to use a single pulse that would satisfy the following conditions : (i) the duration should be long enough to maximize the population transfer (i.e. the alignment), though not too long in order to preserve the revivals of the wave packet, (ii) the intensity should be set below the saturation of ionization. Another possibility is to employ a train of short pulses [24], or to make use of self learning evolutionary algorithm in order to design more sophisticated pulse that would maximize the degree of alignment [25].

\section{Acknowledgments}

This work is supported in part by the European Community's Human Potential Program under contract MTKD-CT-2004-517145 (X-HOMES) and the Ultraviolet Laser Facility (ULF) operating at FORTH-IESL (contract no. HPRI-CT-2001-00139).

\section{Appendix. Estimation of the ionization saturation threshold of OCS molecules based on high order harmonic generation}

Concerning the measurement of the spectrum of the harmonics generated by OCS molecules, the experimental setup of reference [8] has been used. The output from the delay line pump 70 fs laser beam was focused onto the pulsed OCS gas jet where the harmonics where generated. A $0.1 \pm 0.02 \mu \mathrm{m}$ thick Al filter allowed for selecting the bandwidth of $9^{\text {th }}$ to $15^{\text {th }}$ harmonics and for blocking the fundamental laser beam. A 
tungsten coated spherical mirror with $5 \mathrm{~cm}$ focal length focused the XUV beam onto a second gas jet filled with Xe gas and the emitted photoelectrons were detected by a TOF electron spectrometer. Figure A1 (a) shows the photoelectron energy spectrum obtained by the harmonic-single-photon ionization of Xe gas. The double peak formation results from the spin-orbit splitting of the $\mathrm{Xe}^{+}$ground state ${ }^{2} P_{3 / 2}(12.13 \mathrm{eV})$ and ${ }^{2} P_{1 / 2}$ $(13.44 \mathrm{eV})$. This spectrum corresponds to the maximum signal obtained indicating the saturation of OCS ionization.
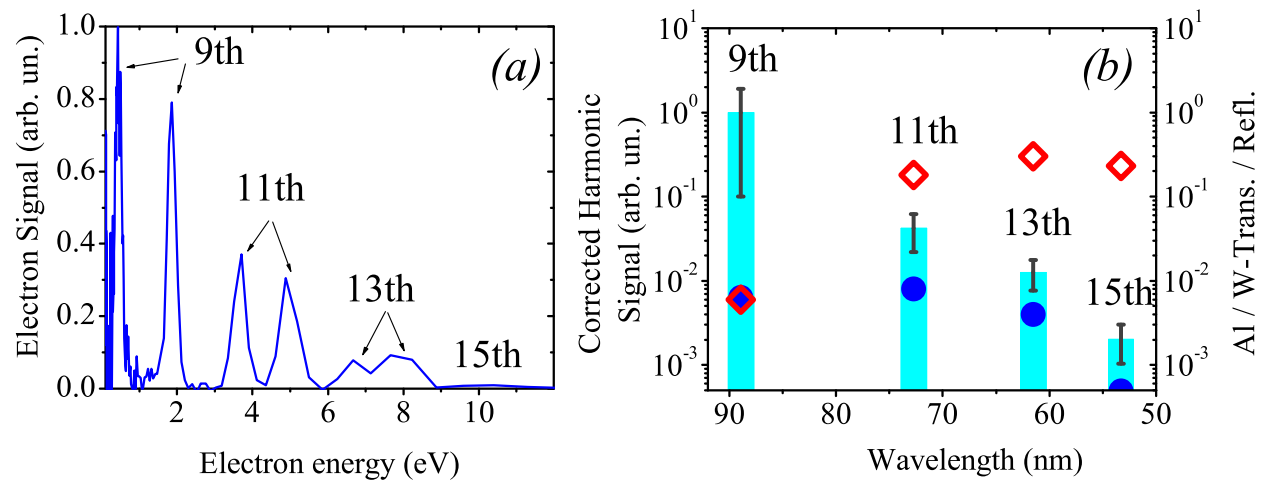

Figure A1. (Color online) (a) The measured energy resolved photoelectron spectrum of Xe due to single-photon ionization by the $9^{\text {th }}-15^{\text {th }}$ harmonics. (b) Filled circles: Measured relative harmonic intensity values. Open diamonds: The transmissionreflectivity of the $\mathrm{Al}$ filter-tungsten mirror in the spectral region of 92 to $50 \mathrm{~nm}$. Vertical bars: The corrected relative harmonic intensities generated in OCS gas jet. Error bars correspond to the uncertainty of the Al filter.

Figure A1 (b) depicts the relative intensities of the harmonics generated in OCS gas jet. The individual harmonic signals (filled circles) have been corrected for the transmission - reflectivity of the Al filter-tungsten mirror (open diamonds). The relative intensity distribution of the harmonics in the corrected harmonic spectrum (vertical bars) indicates that the $11^{\text {th }}$ is the maximum harmonic order corresponding to the cutoff region. According to the cut-off law, $E_{11}=I P-3.17 U_{p}$ (where $E_{11}$ is the photon energy of the $11^{\text {th }}$ harmonic, $I P=11.18 \mathrm{eV}$ the ionization potential of OCS and $U_{p}$ the mean kinetic energy acquired by a free electron oscillating in the driving laser field) an upper limit for the ionization saturation intensity of OCS is estimated to be $30 \mathrm{TW} / \mathrm{cm}^{2}$.

\section{References}

[1] H. Stapelfeldt and T. Seideman. Colloquium: aligning molecules with strong laser pulses. Rev. Mod. Phys., 75:543-57, 2003.

[2] F. Rosca-Pruna and M. J. J. Vrakking. Experimental observation of revival structures in picosecond laser-induced alignment of $\mathrm{i}_{2}$. Phys. Rev. Lett., 87:153902, 2001.

[3] B. Friedrich and D. Herschbach. Alignment enhanced spectra of molecules in intense non-resonant laser fields. Chem. Phys. Lett., 262(1-2):41-6, 1996. 
[4] H. Sakai, C. P. Safvan, J. J. Larsen, K. M. Hilligsoe, K. Hald, and H. Stapelfeldt. Controlling the alignment of neutral molecules by a strong laser field. J. Chem. Phys., 110(21):10235-8, 1999.

[5] H. Sakai, S. Minemoto, H. Nanjo, H. Tanji, and T. Suzuki. Controlling the orientation of polar molecules with combined electrostatic and pulsed, nonresonant laser fields. Physical Review Letters, 90(8):083001/1-4, 2003.

[6] H. Tanji, S. Minemoto, and H. Sakai. Three-dimensional molecular orientation with combined electrostatic and elliptically polarized laser fields. Phys. Rev. A, 72(6):63401-1-4, 2005.

[7] Claude M. Dion, Arne Keller, and Osman Atabek. Optimally controlled field-free orientation of the kicked molecule. Phys. Rev. A, 72(2):023402, 2005.

[8] E. P. Benis, P. Tzallas, L. A. A. Nikolopoulos, M. Kovacev, C. Kalpouzos, D. Charalambidis, and G. D. Tsakiris. Frequency-resolved photoelectron spectra of two-photon ionization of he by an attosecond pulse train. New J. Phys., 8(6):92, 2006.

[9] V. Renard, M. Renard, A. Rouzée, S. Guérin, H. R. Jauslin, B. Lavorel, and O. Faucher. Nonintrusive monitoring and quantitative analysis of strong laser field-induced impulsive alignment. Phys. Rev. A, 70:033420, 2004.

[10] Cho Minhaeng, Du Mei, N. F. Scherer, G. R. Fleming, and S. Mukamel. Off-resonant transient birefringence in liquids. Journal of Chemical Physics, 99(4):2410-28, 1993.

[11] B. Lavorel, O. Faucher, M. Morgen, and R. Chaux. Analysis of femtosecond raman induced polarization spectroscopy (rips) in $\mathrm{n} 2$ and co2 by fitting and scaling laws. J. Raman Spectrosc., 31(1-2):77-83, 2000.

[12] V. Renard, M. Renard, S. Guérin, Y. T. Pashayan, B. Lavorel, O. Faucher, and H. R. Jauslin. Postpulse molecular alignment measured by a weak field polarization technique. Phys. Rev. Lett., 90:153601-4, 2003.

[13] D. S. Elliott and J. F. Ward. Polarizability anisotropies of $\mathrm{co}_{2}, \mathrm{n}_{2} \mathrm{O}$, and ocs from measurements of the intensity-dependent refractive index in gases. Phys. Rev. Lett., 46(5):317, 1981.

[14] A. G. Maki. Microwave spectra of molecules of astrophysical interest, vi. carbonyl sulfide and hydrogen cyanide. J. Phys. Chem. Ref. Data, 3:221-224, 1974.

[15] M. Morgen, W. Price, L. Hunziker, P. Ludowise, M. Blackwell, and Y. Chen. Femtosecond ramaninduced polarization spectroscopy studies of rotational coherences in o2, $\mathrm{n} 2$ and co2. Chem. Phys. Lett., 209(1,2):1-9, 1993.

[16] M. Renard, E. Hertz, S. Guerin, H. R. Jauslin, B. Lavorel, and O. Faucher. Control of field-free molecular alignment by phase-shaped laser pulses. Phys. Rev. A, 72(2):025401, 2005.

[17] H. Ashkenas and F.S. Sherman. The structure and utilization of supersonic free jets in low density wind tunnels. In J.H. de Leeuw, editor, Rarefied Gaz Dynamics, volume II, pages 84-105, New York, 1966. Academic Press.

[18] F. Rosca-Pruna and M. J. J. Vrakking. Revival structures in picosecond laser-induced alignment of i/sub 2/ molecules. ii. numerical modeling. J. Chem. Phys., 116(15):6579-88, 2002.

[19] P. W. Dooley, I. V. Litvinyuk, K. F. Lee, D. M. Rayner, M. Spanner, D. M. Villeneuve, and P. B. Corkum. Direct imaging of rotational wave-packet dynamics of diatomic molecules. Phys. Rev. A, 68(2):23406-23418, 2003.

[20] A. Rouzée, V. Renard, S. Guérin, O. Faucher, and B. Lavorel. Optical gratings induced by fieldfree alignment of molecules. Phys. Rev. A, 75(1):013419-7, 2007.

[21] H. Stapelfeldt. Alignment of molecules by strong laser pulses. Eur Phys J D, 26(1):15-19, 2003.

[22] Vinod Kumarappan, Christer Z. Bisgaard, Simon S. Viftrup, Lotte Holmegaard, and Henrik Stapelfeldt. Role of rotational temperature in adiabatic molecular alignment. J. Chem. Phys., 125(19):194309-7, 2006.

[23] Hasegawa Hirokazu and Ohshima Yasuhiro. Decoding the state distribution in a nonadiabatic rotational excitation by a nonresonant intense laser field. Phys. Rev. A, 74(6):061401, 2006.

[24] C. Z. Bisgaard, M. D. Poulsen, E. Peronne, S. S. Viftrup, and H. Stapelfeldt. Observation of enhanced field-free molecular alignment by two laser pulses. Phys. Rev. Lett., 92(17):173004, 2004 . 
[25] E. Hertz, A. Rouzee, S. Guerin, B. Lavorel, and O. Faucher. Optimization of field-free molecular alignment by phase-shaped laser pulses. Phys. Rev. A, 75(3):031403(R), 2007. 